\title{
THE CONCEPT OF MULTILAYERED NETWORK MODEL FOR 5G NETWORKS
}

\author{
Dávid HRABČÁK*, Ľubomír DOBOŠ** \\ *Department of Electronics and Multimedia Telecommunications, Faculty of Electrical Engineering and Informatics, Technical \\ University of Košice, Boženy Němcovej 32, 04200 Košice, tel. 055/602 2766, E-mail: david.hrabcak@tuke.sk \\ *** Department of Electronics and Multimedia Telecommunications, Faculty of Electrical Engineering and Informatics, Technical \\ University of Košice, Boženy Němcovej 32, 04200 Košice, tel. 055/602 2296, E-mail: lubomir.dobos@ tuke.sk
}

\begin{abstract}
In this paper, actual research of the multilayered networks will be presented. The multilayered networks will become an important phenomenon in an upcoming new generation of $5 G$ networks. In the near future, new network technologies, Internet of Things (IoT) and Smart Cities will reveal new possibilities of $5 G$ networks. These networks along with IoT are considered a promising technology that interconnects different types of networks into one fully functional network. Part of those networks will be also Mobile Ad-Hoc Networks (MANET), Wireless Sensor Networks (WSN) and Fly Ad-Hoc Networks (FANET). The aim of this paper is to present the concept of a multilayered network model consisted of networks mentioned above. The simulations prove, that such a model is able to provide better data rates, delivery time and save some energy due to the lower number of hops.
\end{abstract}

Keywords: Mobile Ad-Hoc Networks, Wireless Sensor Networks, Flying Ad-Hoc networks, Multilayered networks, Concept, Routing

\section{INTRODUCTION}

Upcoming 5G networks bring new opportunities and different view on today's networks. The new phenomenon known as IoT will make the Internet more and more complex, smart and pervasive. All these will lead to complex networks, that will be part of smart homes or even smart cities. To build such a complex network, different types of networks and communication technologies will be interconnected into one functional network. Those networks could be MANET, WSN or FANET, which can be interconnected as a multilayered network model.

The IoT can be simply seen as a sensor network, that collects data sensed from the environment. For these purposes, WSN networks could be applied. Since nodes in WSN are resource-constrained and usually communicates in low data rates, nodes of the MANET network could speed up important data delivery [1]. MANET nodes do not have strict resource constraints and support higher data rates. With the mobility of MANET nodes, important data for processing collected by sensor nodes could be delivered faster. However, the mobility of MANET nodes sometimes causes a network to split into isolated subnetworks. because of this split, nodes in isolated subnetworks are not able to communicate with nodes from other subnetworks. This problem could be solved by flying objects (FO) of FANET, which reconnect subnetworks again. FOs could also collect data from different regions, where WSN and MANET nodes are applied.

Such a multilayered network scenario could be used not only in Smart cities but also as a disaster scenario. To prevent scenarios like earthquake, tsunami, volcano or floods, WSN sensors can be applied in an environment in order to recognize and forecast the occurrence of natural disasters [2]. Data from sensors will be processed by central in the cloud. But when disaster appears, it is important to act quickly. Emergency data needs to be delivered fastest as possible. Since WSN networks are the low data rate networks, MANET and FANET could speed up communication and emergency data delivery. The advantages of
MANET and FANET networks in disaster scenarios are well known. Therefore, multilayered networks will play an important role in the near future with the capability to save a lot of people's lives.

\section{THE CONCEPT OF MULTILAYERED NET- WORK}

The proposed multilayered concept of the network is composed of three layers, that accommodate three different types of wireless networks. Networks used in this multilayered concept are WSN, MANET, and FANET. The main idea is to interconnect three separate networks to one functional network, that is possible to see in Fig 1. For example, in a disaster scenario, WSN sensor that encounters the emergency situation need to deliver its data to the processing center in the cloud as fast as possible. Since the communication in WSN layer is relatively slow, with the multilayered model it uses its nearest sensor gateway to the MANET layer. MANET nodes utilize higher data rates and they are not resource-constrained as WSN sensors. If the nodes of MANET does not have direct connectivity to the processing center in the cloud, emergency data are sent through the MANET gateway node to the FO of FANET network. FOs are able to operate at longer distances in a multi-hop manner. Emergency data are transferred through FANET network to the MANET sub-network with connectivity to the processing center in the cloud. This solution could deliver emergency data faster than WSN network. The multilayered concept could be also used as data harvesting when WSN sensors can be deployed in different environments without direct connectivity to the processing center. The MANET and FANET nodes could collect data from WSN and deliver them. 


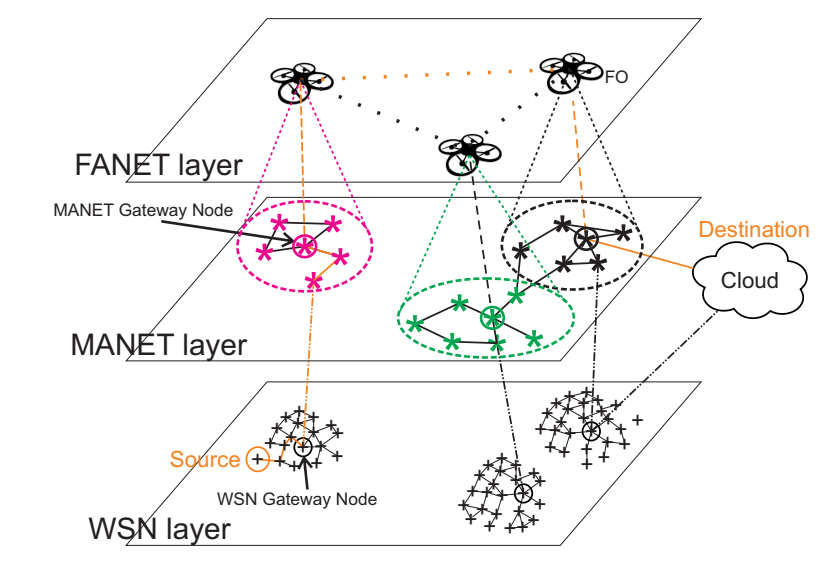

Fig. 1 The Concept of Multilayered Network Routing

\subsection{The WSN network layer}

The first layer consists of low energy wireless sensors of WSN network, where they operate in a multi-hop adhoc manner. Sensors of the WSN network are resourceconstrained devices, that usually operate at low data rates in order to keep power consumption down. Therefore, communication among sensors is realized by IEEE 802.15.4 ZigBee at the link layer, which enables sensors to communicate over distances up to about 10 meters and with maximum transfer data rates of $250 \mathrm{Kbps}$. Newest embedded devices are able to communicate with lower transfer rates of 20 and $40 \mathrm{Kbit} / \mathrm{s}$ [3]. There are two types of sensors in WSN network. First is a regular sensor that collects measured data and communicates with other sensors through standard WSN protocol like [4]. Second are WSN gateway sensors, that are able to communicate with MANET nodes and processing center in the cloud. Gateway sensor uses two protocol stack, IEEE 802.15.4 for sensors communication and IEEE 802.11 for MANET communication [5]. To connect gateway sensor nodes to the cloud on the Internet, 6LowPan protocol is used between the routing layer and MAC layer [6,7].

\subsection{The MANET network layer}

The second layer consists of MANET nodes, that could be wireless devices like smartphones, laptops, PDAs and other. These nodes are not strictly resource-constrained and enable higher data rates through the IEEE 802.11 link layer standard. As a compatible routing protocol, IPv6 enabled DSR protocol [8] is used in order to keep compatibility with WSN layer. Every MANET nodes are therefore enabled to communicate with WSN gateway nodes. To enable MANET nodes to communicate with FANET network, MANET network needs to be divided into clusters. The division into clusters can be performed by Particle Swarm Optimization algorithm (PSO) [9]. Each cluster chooses its cluster head, that act as a gateway to the FANET. Both MANET gateway and FANET FO utilizes the IEEE 802.11 standard.

\subsection{The FANET network layer}

The third layer consist of FO, also knowns as unmanned aerial vehicles (UAVs). Those FOs are flying above MANET and WSN networks. Nodes of the MANET network are divided into the clusters. FOs covers these clusters in order to communicate with MANET cluster head, that act as MANET gateway. FO could use IEEE 802.11 standard, that allows communication with MANET nodes and among FOs. Communication among FOs is provided by FANET routing protocols, for example, a hybrid routing protocol for the A2A links like AODV or OLSR [10]. Since FOs are significantly higher than the MANET and WSN nodes, radio range distances are much higher (up to $250 \mathrm{~m}$ ) because of direct sight without any obstacles. With the utilization of two protocol stack, FOs could also use other communication standards like IEEE 802.16 WiMAX. With WiMAX, FOs are able to communicate above $10 \mathrm{MBps}$ with a distance up to $10 \mathrm{~km}[11]$.

All three layers are working independently except for emergency situations. Interconnection and inter-layer communication are based on network gateways. Sensors of the WSN network chooses its gateway nodes, which operates with a higher energy level than the non-gateway node. MANET nodes choose their gateway nodes as well. Based on the location of the MANET gateway node, FOs of FANET are able to focus its flying plan and antennas. Therefore, routing protocols need to be carefully evaluated in future research.

\section{SIMULATIONS}

In this section, the simulations of the multilayered network model will be described. The main focus of the simulations was to show advantages of the multilayered network model over conventional WSN network. Therefore, the simulation scenario consists of two networks. The first simulated network was WSN network consisted of 400, 500 and 600 sensor nodes. These nodes were placed on an area of 200 x 200 meters. In this network, an energy-aware QoS routing protocol for wireless sensor networks was used [4]. For this reason, the gateway sensors have been selected from conventional sensors so that each sensor is able to reach the nearest gateway sensor at least at 2 hops. The source sensor node always looking for the nearest gateway sensor. Then the data are transported through the gateway sensors by Dijkstra shortest path algorithm to the Access Point, which is basically the gateway to the Cloud. The shortest path is calculated based on the highest data rates on the links. For each link, the data rate value is randomly generated from the range of $30 \mathrm{Kbps}+-50 \%$. The radio range of WSN nodes was set to 10 meters. The example of this simulation is possible to see on fig 2 


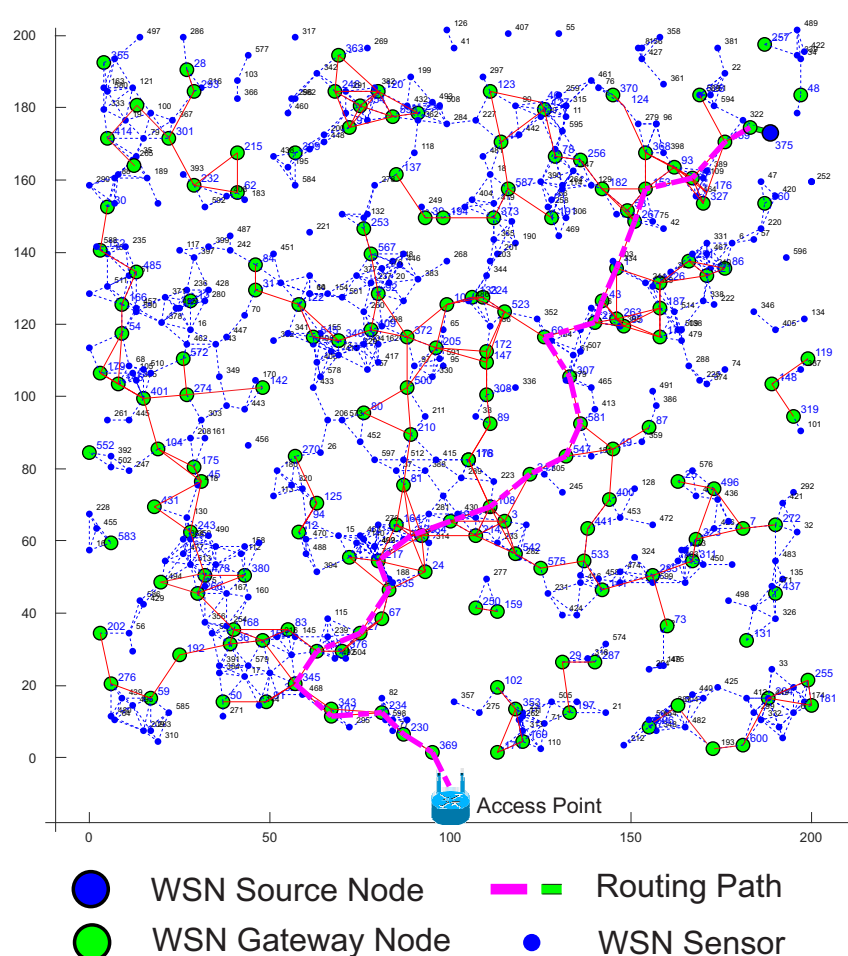

Fig. 2 The Example of conventional WSN network simulation

The second network was a multilayered model consisted of WSN, MANET and FANET network. WSN layer consisted of 400, 500 and 600 sensor nodes in the area of 200x200 meters. The 12 gateway sensors were chosen from conventional sensors in order to cover the area evenly. The gateway sensors are using a second protocol stack, so they are able to communicate with MANET nodes on the second layer. Radio ranges and data rates were chosen the same as in the first conventional WSN scenario. In all simulations of the multilayered network model, 50 MANET nodes were used with the 40-meters radio range and with data rates randomly generated from the range of $100 \mathrm{Mbps}+-50 \%$. If the WSN source node sends the urgent data, it looks for the nearest gateway sensor. If the Access Point to the cloud is not directly connected, the gateway sensor sends data to the nearest MANET node. If the Access Point is connected to the MANET network, the data are sent to the Access Point via Dijkstra shortest path algorithm based on data rates on the links. If the Access Point is not connected to the MANET network and there is a FANET network available, data are sent to the FANET layer. In the MANET layer, the PSO clustering algorithm is performed in order to divide MANET nodes to clusters, that are later covered by drones. In each cluster, the cluster head is selected based on the highest node degree. The cluster head is acting as a MANET gateway node to the FANET. FANET drones in this simulation scenario are communicating through IEEE $802.11 \mathrm{n}$ with a radio range of 200 meters and $250 \mathrm{Mbps}+-$ $50 \%$ data rate on links. After conventional MANET node is not able to find Access Point in the MANET network, it looks for the nearest MANET cluster head and it sends data to it. MANET cluster head that sends data to the drone, which routes data to the nearest drone via Dijkstra short- est path algorithm based on data rates on links, that covers MANET network with connection to the Access Point. Data are then directed to the MANET cluster head of the MANET network with connection to do Access Point and then through MANET nodes to the destination. The example of multilayered model simulation is possible to see in Fig 3. Detailed situation is possible to see in Fig 4 and Fig 5 In both simulation scenarios, $100 \mathrm{~Kb}$ of data was sent from the source WSN node to Access Point.

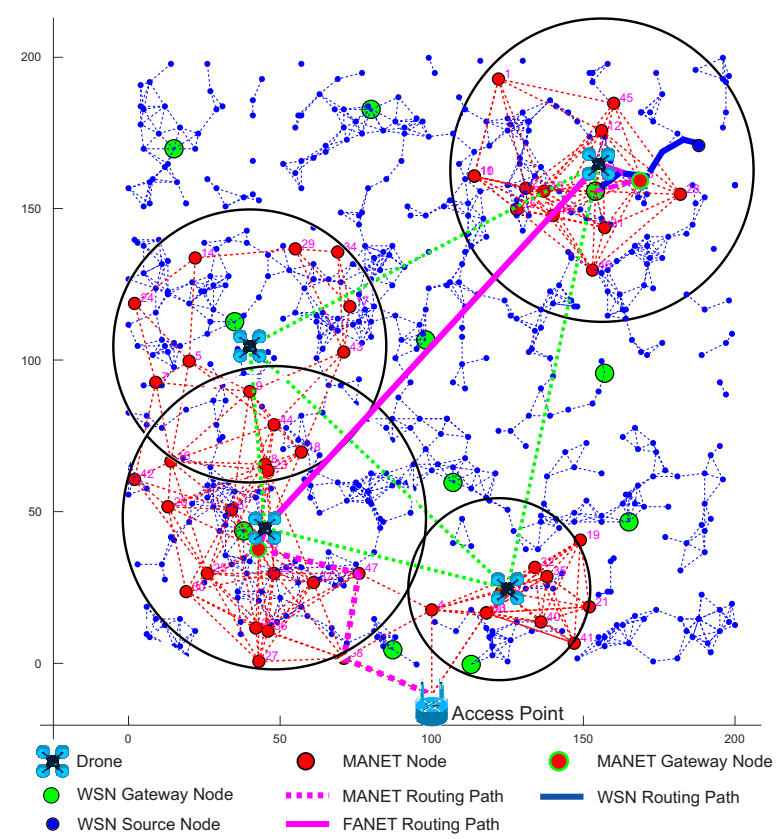

Fig. 3 The example of multilayered network model simulation.

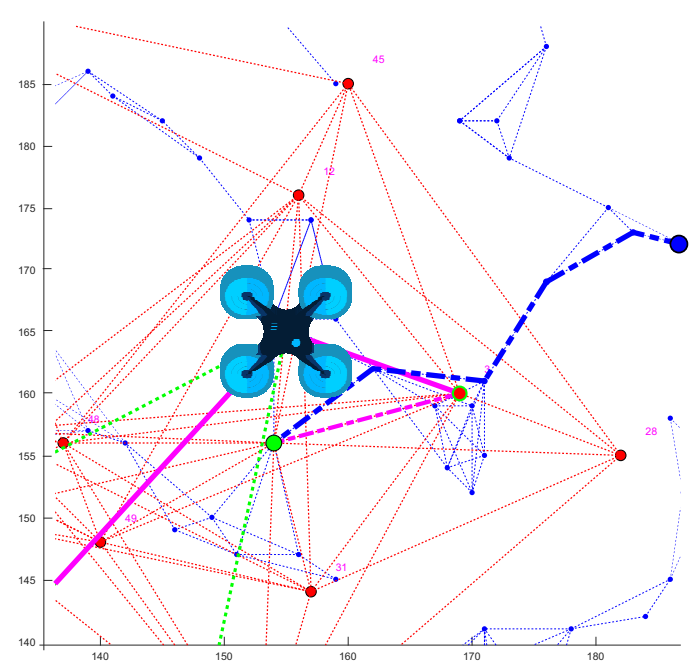

Fig. 4 Detailed look at multilayered network model simulation. 


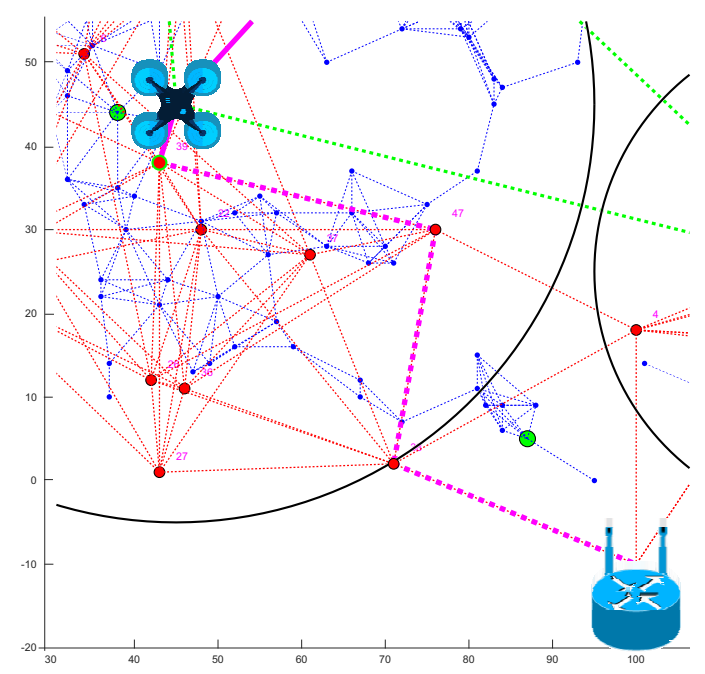

Fig. 5 Detailed look at multilayered network model simulation.

\subsection{Simulation Results}

In this section will be presented results based on the described simulations. In all simulated scenarios number of WSN nodes changes from 400,500 to 600 with the same positions of nodes in all simulations. The presented results are average numbers calculated from 100 simulation runs.

The first result is oriented on average delivery time in Fig 6. It is possible to see, that multilayered (WSN MANET FANET) network model obtains dramatically better time values than convention WSN network in all scenarios.

\section{Average delivery time}

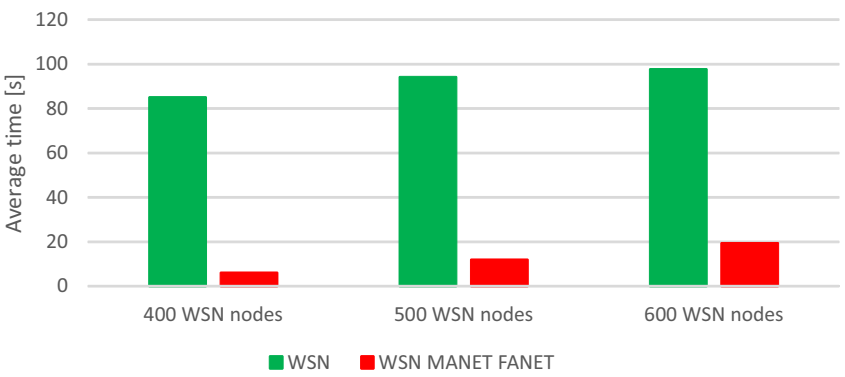

Fig. 6 Average delivery time of $100 \mathrm{~Kb}$ message

In the second result (Fig 7), that is oriented on the average number of hops is possible to see the same behavior. The number of hops in the multilayered network model is better in all scenarios. Since the energy for each re-routing and hop is needed, it is also possible to assume, that with a lower number of hops for the multilayered network is possible to save some amount of energy.
Average number of hops from source to destination

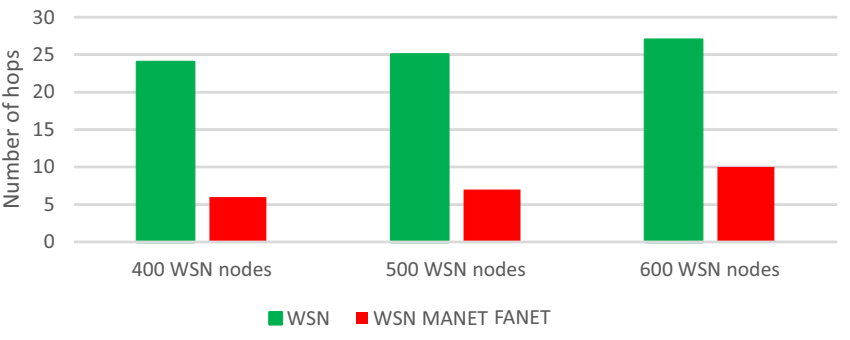

Fig. 7 Average number of hops on the path from source node to AP.

The third result is presented in the form of table 1. In this table is possible to see average data rates on the routing paths in Mbps. In conventional WSN network, data rates are around 0,03 Mbps, whereas in multilayered network model data rates are significantly higher, from 49 to $83 \mathrm{Mbps}$. These data rates are also helping the multilayered model to achieve better delivery times.

Table 1 Average data rate (Mbps) of $100 \mathrm{~Kb}$ data delivery.

\begin{tabular}{|l|r|r|}
\hline Average Data Rate [Mbps] & WSN & WSN MANET DRONET \\
\hline 400 WSN nodes & 0,0293 & 83,03 \\
\hline 500 WSN nodes & 0,0302 & 64,67 \\
\hline 600 WSN nodes & 0,0307 & 49,94 \\
\hline
\end{tabular}

\section{CONCLUSION}

In this paper, the concept of multilayered network routing was proposed. Interconnection of different types of networks like MANET, WSN, and FANET will be a common phenomenon of upcoming $5 \mathrm{G}$ networks. The concept could be utilized in many different areas of the new generation network and also useful in disaster scenarios. The concept is right now under the deep theoretical scope and the further simulations are built on the basis of acquired knowledge. Based on actual simulations is possible to say, that the multilayered network model is able to provide multiple advantages over conventional WSN network when urgent data needs to be delivered quickly or the network is disconnected into separate islands. In future research, the concept will be implemented and tested in the real world with real devices.

\section{ACKNOWLEDGEMENT}

This work has been performed partially in the framework of the Ministry of Education of Slovak Republic under research projects VEGA 1/0492/18, KEGA 046TUKE4/2018 and APVV-17-0208.

\section{REFERENCES}

[1] JABBAR, W. A. - SAAD, W. K. - ISMAIL, M.: "MEQSA-OLSRv2: A Multicriteria-Based Hybrid 
Multipath Protocol for Energy-Efficient and QoSAware Data Routing in MANET-WSN," Convergence Scenarios of IoT. IEEE Access, 6, 76546-76572, 2018.

[2] ERDELJ, M. - NATALIZIO, E.: "UAV-assisted disaster management: Applications and open issues," Int. J. Advanced Networking and Applications, 10(03), 3833-3842, 2018.

[3] "IEEE Standard for Local and metropolitan area networks-Part 15.4: Low-Rate Wireless Personal Area Networks (LR-WPANs)," in IEEE Std 802.15.42011 (Revision of IEEE Std 802.15.4-2006), pp.1314, 2011.

[4] AKKAYA, K. - YOUNIS, M.: "An energy-aware QoS routing protocol for wireless sensor networks, In Distributed Computing Systems Workshops, 2003. Proceedings," 23rd International Conference on (pp. 710715). IEEE, 2003.

[5] GAST, M.: "802.11 wireless networks: the definitive guide," OReilly Media Inc Pub (2005): 656, 2005.

[6] KUSHALNAGAR, N. - MONTENEGRO, G. SCHUMACHER, C.: "IPv6 over low-power wireless personal area networks (6LoWPANs): overview, assumptions, problem statement, and goals," (No. RFC 4919), 2007.

[7] KIM, E. - KASPAR, D. - GOMEZ, C. - BORMANN,C.: "Problem statement and requirements for IPv6 over low-power wireless personal area network (6LoWPAN) routing,"(No. RFC 6606), 2012.

[8] LIU, Q. - QIN, H.: “ Implementation and Improvement of DSR in Ipv6," Procedia Engineering, 29, 716$720,2012$.

[9] LOSCRI, V. - NATALIZIO, E. - MITTON, N.: "Performance evaluation of novel distributed coverage techniques for swarms of flying robot," In Wireless Communications and Networking Conference (WCNC), 2014 IEEE (pp. 3278-3283).

[10] DANIEL, K. - WIETFELD, C.: "Using public network infrastructures for UAV remote sensing in civilian security operations," DORTMUND UNIV (GERMANY FR), 2011.

[11] GHOSH, A. - WOLTER, D. R. - ANDREWS, J. G. - CHEN, R.: "Broadband wireless access with WiMax/802.16: current performance benchmarks and future potential," IEEE communications magazine, 43(2), 129-136, 2015.

Received September 30, 2019, accepted November 11, 2019

\section{BIOGRAPHIES}

Dávid Hrabčák graduated (Ing.) in 2016 at the department of Mathematics and Theoretical Informatics of the Faculty of Electrical Engineering and Informatics at Technical University in Košice. In 2017 he begins his $\mathrm{PhD}$ study in the field of MANET networks and cognitive radio at the department of Electronics and Multimedia Telecommunications; His thesis title will be entitled as "Adaptive Routing Protocols for CR MANET".

Ľubomír Doboš was born on 12. 09. 1991 works as an Associate Professor at the Department of Electronics and Multimedia Telecommunications, the Faculty of Electrical Engineering and Informatics of the Technical University of Koice. His scientific research areas are broadband information and telecommunication technologies, multimedia systems, telecommunications networks and services, 5G networks, localization algorithms. 